\title{
Étude à méthode mixte d'une intervention de soutien prodiguée par les pairs aux patients nouvellement diagnostiqués d'une tumeur cérébrale primitive
}

\author{
par Douglas Ozier et Rosemary Cashman
}

\section{ABRÉGÉ}

Objectif: L'objectif de cette étude pilote portait sur les répercussions d'une intervention conçue pour améliorer la qualité de vie des patients nouvellement diagnostiqués d'une tumeur cérébrale primitive. L'intervention comprenait une seule rencontre structurée entre des patients nouvellement diagnostiqués d'une tumeur cérébrale primitive et des patients bénévoles qui avaient déjà souffert d'une tumeur cérébrale primitive et qui avaient reçu une formation dans le cadre de cette intervention.

Méthodologie: Deux bénévoles ont eu une rencontre individuelle avec un total de dix patients nouvellement diagnostiqués d'une tumeur cérébrale primitive. Une combinaison de questionnaires et d'entretiens a été utilisée pour étudier les répercussions de l'intervention sur les nouveaux patients et les bénévoles.

Résultats: L'intervention s'est montrée d'une valeur substantielle pour les deux groupes de participants. L'analyse a révélé que les patients nouvellement diagnostiqués en ont retiré de nombreux avantages, $y$ compris ceux-ci: espoir accru, conseils précieux, savoir exactement de quoi il en retourne, solitude atténuée et prise de

\section{AU SUJET DES AUTEURS}
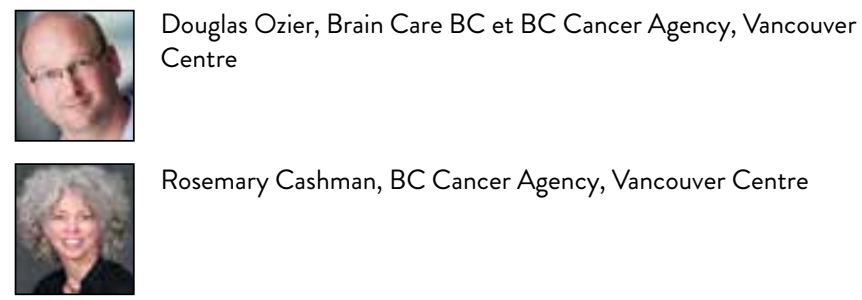

Douglas Ozier, Brain Care BC et BC Cancer Agency, Vancouver Centre

Rosemary Cashman, BC Cancer Agency, Vancouver Centre

Auteur à qui adresser la correspondance : Dr Douglas Ozier (Ph.D. en psychologie du counseling), clinicien chercheur au Brain Care BC et au programme provincial d'oncologie psychosociale du BC Cancer Agency, \#653-750 W. Broadway St., Vancouver (Colombie-Britannique) V5W $1 \mathrm{H} 5$

Téléphone : 604-877-6000, poste 672185, téléc. : 604-877-6249; dozier@bccancer.bc.ca

Deuxième auteure: Rosemary Cashman, inf. aut., M.A., M.Sc. (A), IP (A), infirmière praticienne au BC Cancer Agency, \#6680-600 W. 10th Ave., Vancouver (Colombie-Britannique) V5Z 4E6

Téléphone : 604-877-6072; rcashman@bccancer.bc.ca

Conflits d'intérêts et sources de financement: Cette recherche est sans financement et nous n'avons aucun conflit d'intérêts à déclarer.

DOI: $10.5737 / 23688076262112121$ conscience sur ce qui importe vraiment. Seuls des effets indésirables mineurs et de légers problèmes ont été signalés.

Conclusions: Les constats fournissent des données probantes initiales selon lesquelles l'intervention élaborée peut représenter un moyen sécuritaire et utile d'améliorer le bien-être psychosocial chez les patients nouvellement diagnostiqués d'une tumeur cérébrale primitive.

La réalisation d'autres études concernant le potentiel de soutien individuel par des pairs pour les patients atteints d'une tumeur cérébrale primitive constitue une priorité importante en matière de recherche.

Mots clés : cancer; tumeur cérébrale; soutien par les pairs; qualité de vie; bénévole

\section{INTRODUCTION}

$\mathbf{R}$ ecevoir un diagnostic de tumeur cérébrale primitive est un événement dévastateur. L'impact de ce diagnostic se reflète dans le fait que les patients atteints d'une tumeur cérébrale primitive présentent une importante détérioration de leur qualité de vie (Taphoorn, Sizoo et Bottomley, 2010), un niveau très élevé de détresse existentielle (Pelletier, Verhoef, Khatri et Hagen, 2002) et des taux de dépression qui sont trois fois plus élevés que ceux que l'on retrouve parmi la population atteinte de cancer en général (Wellisch, Kaleita, Freeman, Cloughesy et Goldman, 2002). Il existe un besoin évident en ce qui concerne l'élaboration et la validation d'interventions psychosociales pouvant aider à améliorer la qualité de vie et à diminuer la détresse chez les patients atteints d'une tumeur cérébrale primitive.

Pour répondre à ce besoin, notre équipe de recherche a consulté les membres de notre Conseil consultatif des patients et des familles (CCPF), un organe bénévole formé de patients atteints d'une tumeur cérébrale primitive et d'aidants naturels pour les familles qui formule des observations au sujet du programme de soins en neuro-oncologie du British Columbia Cancer Agency (BCCA). Les membres du CCPF ont recommandé l'élaboration d'un programme pilote pour les patients nouvellement diagnostiqués d'une tumeur cérébrale primitive au moyen d'une rencontre individuelle avec un « ancien » patient qui serait formé pour offrir du soutien et de l'information. Les nouveaux patients participants seraient jumelés aux anciens patients bénévoles selon qu'ils aient une tumeur d'un grade élevé (III et IV) ou faible (II). 
Une revue de la documentation a indiqué de nombreuses données probantes pour appuyer ce projet. Premièrement, les résultats de recherche permettent de penser que même si les survivants du cancer qui font partie d'un groupe de soutien en retirent des avantages, seul un faible pourcentage de ces survivants a déjà fait partie d'un tel groupe (Grande, Myers et Sutton, 2006). Par exemple, un groupe d'auteurs (Sherman, Pennington, Simonton, Latif, Arent et Farley, 2008) a indiqué que seulement $8 \%$ des 425 survivants de cancer interrogés avaient participé à un groupe de soutien. Par conséquent, les interventions de soutien individuelles par des pairs sembleraient offrir une solution de rechange aux groupes de soutien, attirant peut-être les patients atteints de cancer qui pourraient ne pas se sentir à l'aise d'accéder au soutien dans un contexte de groupe. D'ailleurs, nous nous sommes demandé si l'offre d'une interaction positive par des pairs au début de la maladie pouvait aider les patients nouvellement diagnostiqués d'une tumeur cérébrale à accéder plus activement à d'autres services de soutien, notamment des groupes de soutien.

Deuxièmement, une revue de 19 interventions de soutien individuelles menées dans un contexte oncologique a indiqué que ces programmes ont tendance à être bien reçus et qu'ils présentent des avantages, notamment pour ce qui est de l'amélioration du bien-être ou de la diminution de l'anxiété (Macvean, White et Sanson-Fischer, 2008, p. 22). Par exemple, un groupe d'auteurs (Dunn, Stegingia, Occhipinti et Wilson, 1999) a mené des recherches sur les répercussions du programme « Surmonter le cancer », lequel organise des rencontres entre les survivantes du cancer du sein ayant reçu une formation et les patientes qui ont récemment subi une mastectomie. Les nouvelles patientes reçoivent une seule visite brève de leur bénévole. Malgré le caractère bref de ces interactions, en moyenne, les 245 nouvelles patientes atteintes de cancer du sein ont trouvé que cette intervention était « très utile » et qu'elles étaient « beaucoup moins anxieuses » après les rencontres.

Troisièmement, l'adaptation au diagnostic de cancer survient à mesure que le temps passe et à l'aide d'efforts. L'acquisition d'une certaine maîtrise a été mentionnée en tant qu'élément important faisant partie de l'ajustement à une maladie possiblement mortelle (Hagopian, 1993). On a émis l'hypothèse selon laquelle les conseils prodigués aux nouveaux patients seraient non seulement utiles pour ces derniers, mais qu'ils pourraient également favoriser le sentiment de maitrise chez les patients intervenants et, par conséquent, renforcer leur force psychologique et leur capacité d'adaptation.
Enfin, malgré la promesse claire des interventions de soutien individuelles des pairs en psycho-oncologie, relativement peu de recherches ont été effectuées dans ce domaine. Ceci a amené les auteurs du domaine à exiger davantage de recherches (Macvean, White et Sanson-Fischer, 2008; Hoey, Ieropoli, White et Jefford, 2008).

Dans cet article, nous dressons un rapport sur les répercussions de l'intervention de soutien par des pairs que nous avons dirigée de juin à novembre 2012. Notre recherche a porté sur ces questions:

- Les nouveaux patients participants ont-ils retiré des avantages ou ont-ils eu des résultats défavorables?

- Y a-t-il eu des avantages ou des résultats défavorables ressentis par les patients intervenants bénévoles?

- Quels autres renseignements obtenus de cette étude pourraient conséquemment améliorer l'intervention?

\section{MÉTHODOLOGIE}

\section{Participants}

Patients intervenants bénévoles. Quatre patients bénévoles ayant surmonté une tumeur semblable ont été recrutés à partir de la communauté vancouvéroise des patients atteints d'une tumeur cérébrale; tous étaient des membres actifs de notre CCPF et avaient terminé leur traitement initial pour une tumeur cérébrale primitive (voir tableau 1).

Le premier auteur a donné 15 heures de formation individuelle à chaque patient intervenant bénévole pendant six semaines. Les séances de formation portaient sur la clarification du rôle, la gestion émotionnelle et l'utilisation intensive des jeux de rôles pour l'intégration des habiletés pertinentes. Des protocoles concernant diverses questions de limites possibles (par exemple, la présence d'idées suicidaires et la « critique contre les médecins ») ont aussi été créés.

Trois patients intervenants bénévoles ont suivi la formation. Malheureusement, la quatrième bénévole, Ann, a présenté une récidive de sa tumeur après avoir reçu sa première séance de formation et elle s'est retirée de l'étude.

Nouveaux patients participants. À titre d'évaluation initiale des patients nouvellement diagnostiqués d'une tumeur cérébrale, notre travailleuse sociale en neuro-oncologie a invité tous les patients admissibles à participer à l'étude lors de leur première consultation à la clinique BCCA du Vancouver Centre. Les critères de participation étaient: un nouveau diagnostic de gliome de grade II, III ou IV; l'aisance à s'exprimer

Tableau 1: Patients intervenants bénévoles

\begin{tabular}{|l|l|l|l|l|l|l|}
\hline Pseudonyme & Sexe & Âge & $\begin{array}{l}\text { Appartenance } \\
\text { ethnique }\end{array}$ & Profession & Diagnostic & $\begin{array}{l}\text { Années } \\
\text { post-diagnostic }\end{array}$ \\
\hline Yves & M & 38 & Race blanche & Scientifique & Glioblastome/grade 4 & 9 \\
\hline Mike & M & 39 & Race blanche & $\begin{array}{l}\text { Ancien avocat/Conseiller pour les } \\
\text { étudiants }\end{array}$ & $\begin{array}{l}\text { Astrocytome de type } \\
\text { anaplastique/grade 3 }\end{array}$ & 4 \\
\hline Arnold & M & 66 & Race blanche & Universitaire semi-retraité & Oligodendrogliome/grade 2 & 6 \\
\hline Ann & F & 41 & Race blanche & Ancienne ergothérapeute/mère au foyer & Oligodendrogliome/grade 2 & 4 \\
\hline
\end{tabular}


en anglais; et un indice $\geq 70$ selon l'échelle de Karnofsky. Le premier auteur a ensuite effectué des appels de dépistage à tous les nouveaux patients qui se sont montrés intéressés à participer à l'étude. Après avoir donné son consentement éclairé, chaque nouveau patient participant a rencontré un patient intervenant bénévole jusqu'à concurrence de 10 rencontres individuelles.

Le recrutement s'est déroulé du $1^{\text {er }}$ avril au 29 août 2012. Pendant cette période, 31 patients anglophones ayant été nouvellement diagnostiqués d'un gliome de haut grade se sont présentés pour des traitements au Vancouver Centre. Parmi ceux-ci:

- 8 ont été exclus en raison d'un état fonctionnel inadéquat;

- 23 participants potentiels ont été invités à recevoir un appel de dépistage;

- 13 de ces 23 personnes ont refusé l'offre;

- les 10 patients intéressés ont été inscrits avec succès (voir le tableau 2).

Pendant la période de régularisation, aucun nouveau patient atteint d'une tumeur cérébrale de faible grade répondant aux critères d'inclusion ne s'est présenté à la clinique. Par conséquent, pour maintenir notre objectif d'associer des patients intervenants bénévoles avec de nouveaux patients participants selon le grade de la tumeur, chacune de ces rencontres a été effectuée par ces patients bénévoles qui avaient reçu un diagnostic de gliome de haut grade (Mike: 6 rencontres; Yves : 4 rencontres).

\section{Intervention}

L'intervention comprenait une rencontre privée d'une heure. Les rencontres avaient lieu dans une salle de réunion à la clinique BCCA du Vancouver Centre, de quatre à huit semaines après le diagnostic initial des patients nouvellement diagnostiqués. Les patients intervenants bénévoles ont structuré les rencontres pour y inclure des introductions, du temps pour que les nouveaux patients puissent discuter de sujets libres ou poser des questions, ainsi qu'une brève période à la fin pendant laquelle les patients intervenants bénévoles ont passé en revue une liste des ressources de soutien pertinentes. Les patients intervenants bénévoles avaient reçu une formation concernant l'utilisation d'une liste de questions au cas où les nouveaux patients participants sembleraient hésiter à poser leurs propres questions. Un exemple type de la liste était: « Beaucoup de nouveaux patients sont inquiets à propos des effets secondaires du traitement, est-ce votre cas? ». Dans l'ensemble, les patients intervenants bénévoles ont mis l'accent sur l'écoute active, la prestation de soutien émotionnel et le partage des expériences personnelles réussies à titre de survivant. Les patients intervenants bénévoles ont été expressément formés à ne jamais offrir de conseil médical sous quelque forme que ce soit. Les nouveaux patients participants ont rempli les questionnaires immédiatement après l'intervention.

\section{Mesure psychométrique}

Le questionnaire de l'étude, de type Likert, une mesure d'auto-évaluation, se composait de 10 questions et était adapté du questionnaire Post-Meeting Participant Response (Ashbury, Cameron, Mercer, Fitch et Nielsen, 1998). Il visait à évaluer quantitativement les réactions des nouveaux patients participants face à l'intervention.

\section{Entrevues de suivi}

Le premier auteur a rencontré les nouveaux patients participants une semaine après l'intervention lors d'un entretien de suivi enregistré sous forme numérique. Six semaines plus tard, le premier auteur a communiqué avec les nouveaux patients participants par téléphone pour un deuxième entretien. Le premier auteur a également effectué des entretiens auprès des patients intervenants bénévoles à la fin de la période de la collecte de données. Tous les entretiens comprenaient des questions quantitatives et évaluatrices, des questions fermées et des questions exploratrices ouvertes (voir l'annexe A pour connaître quelques-unes des questions posées).

\section{Supervision et soutien des patients intervenants bénévoles}

Le premier auteur a rencontré chaque patient intervenant bénévole de manière individuelle après que le patient intervenant bénévole ait effectué deux séances avec les nouveaux patients participants. Ces rencontres de supervision d'une heure abordaient la résolution de problèmes, l'encadrement et le soutien émotionnel.

\begin{tabular}{|c|c|c|c|c|c|}
\hline Nom & Âge & Sexe & Appartenance ethnique & État civil & Grade du gliome \\
\hline Gerry & 48 & $M$ & Race blanche & Marié & 3 \\
\hline Gary & 54 & $M$ & Asiatique & Marié & 4 \\
\hline Jake & 54 & $M$ & Race blanche & Marié & 3 \\
\hline Bradley & 57 & $M$ & Race blanche & Marié & 4 \\
\hline Dwayne & 65 & $M$ & Race blanche & Marié & 4 \\
\hline Shawn & 68 & $M$ & Race blanche & Marié & 4 \\
\hline Shirley & 47 & $\mathrm{~F}$ & Race blanche & Conjointe de fait & 3 \\
\hline
\end{tabular}




\section{ANALYSE DES DONNÉES}

Analyse quantitative. Les questions d'entretien servant à l'évaluation et les réponses au questionnaire ont été analysées à l'aide de statistiques descriptives.

Analyse qualitative. L'analyse des entretiens a suivi l'approche générale de l'analyse qualitative décrite par Vilhauer (2009). Tout d'abord, tous ces entretiens ont été transcrits par des professionnels. Le premier auteur a ensuite lu et relu les transcriptions des entretiens initiaux des nouveaux patients participants pour établir toutes les parties de ces transcriptions qui étaient liées à l'un des domaines de recherche définis précédemment: avantages, effets indésirables, sujets importants et suggestions d'amélioration. Ce procédé a révélé que les questions liées aux domaines que sont les sujets importants et les suggestions d'amélioration ont entraîné des réponses assez directes. Les réponses de ces catégories n'ont donc pas été analysées de manière thématique, mais sont plutôt énumérées sous forme de liste accompagnée des comptes de fréquence. De plus, l'analyse a indiqué que seules deux réactions indésirables ont été signalées. Par conséquent, chacune de ces réactions est discutée de manière individuelle. Pour leur part, les réponses des nouveaux patients participants au sujet des avantages dérivés ont suscité un ensemble varié de données narratives. Ainsi, ces données ont été codées en fonction des thèmes contenus dans les unités de sens. La méthode de comparaison constante (Strauss et Corbin, 1990) a ensuite été utilisée pour grouper ces données dans les thèmes en émergence et, s'il y a lieu, dans les sous-thèmes.

Le procédé décrit a également été utilisé sur les données des deuxièmes entretiens avec les nouveaux patients participants. Comme dans le cas des données de la première série, seules les réponses liées aux avantages perçus ont donné lieu à des données soumises à l'analyse thématique. La comparaison des données liées aux avantages entre ces deux points temporels a révélé qu'aucun nouveau thème lié aux avantages n'est ressorti des deuxièmes entretiens. Les deux ensembles de données liées aux avantages ont donc été fusionnés pour les analyses subséquentes.

Une fois que toutes les données liées aux avantages avaient été codées et assignées à un thème, un codeur naïf a trié de nouveau un échantillon aléatoire représentant $30 \%$ de ces données (Wimmer et Dominick, 1991). Après ce tri, les thèmes se sont précisés. Ce procédé a été répété jusqu’à ce qu’un coefficient de concordance Kappa d'au moins 0,7 soit atteint entre les codeurs, ce qui représente un «bon » niveau de concordance (Altman, 1991). On a évalué qu'un thème (espoir augmenté) contenait des sous-thèmes. Par conséquent, le procédé de retriage et d'amélioration décrit a été répété pour les sousthèmes à l'aide d'un autre codeur naïf, jusqu'à ce qu'un coefficient Kappa de 0,7 ou plus soit atteint.

En ce qui concerne les entretiens des patients intervenants bénévoles, le procédé d'analyse a commencé également par la transcription professionnelle des deux entretiens. Le chercheur principal a ensuite déterminé quelles parties des transcriptions étaient liées à l'un des domaines de recherche précédemment décrits: avantages, effets indésirables, problèmes et réactions à la formation et au soutien.

\section{Déontologie}

L'autorisation déontologique de cette étude a été obtenue du BC Cancer Agency Research Ethics Board. Le consentement éclairé a été obtenu de tous les participants compris dans l'étude.

\section{RÉSULTATS}

Réponses au questionnaire (Tableau 3).

Tableau 3 : Réponses des nouveaux patients participants au questionnaire « Réactions à l'intervention " *

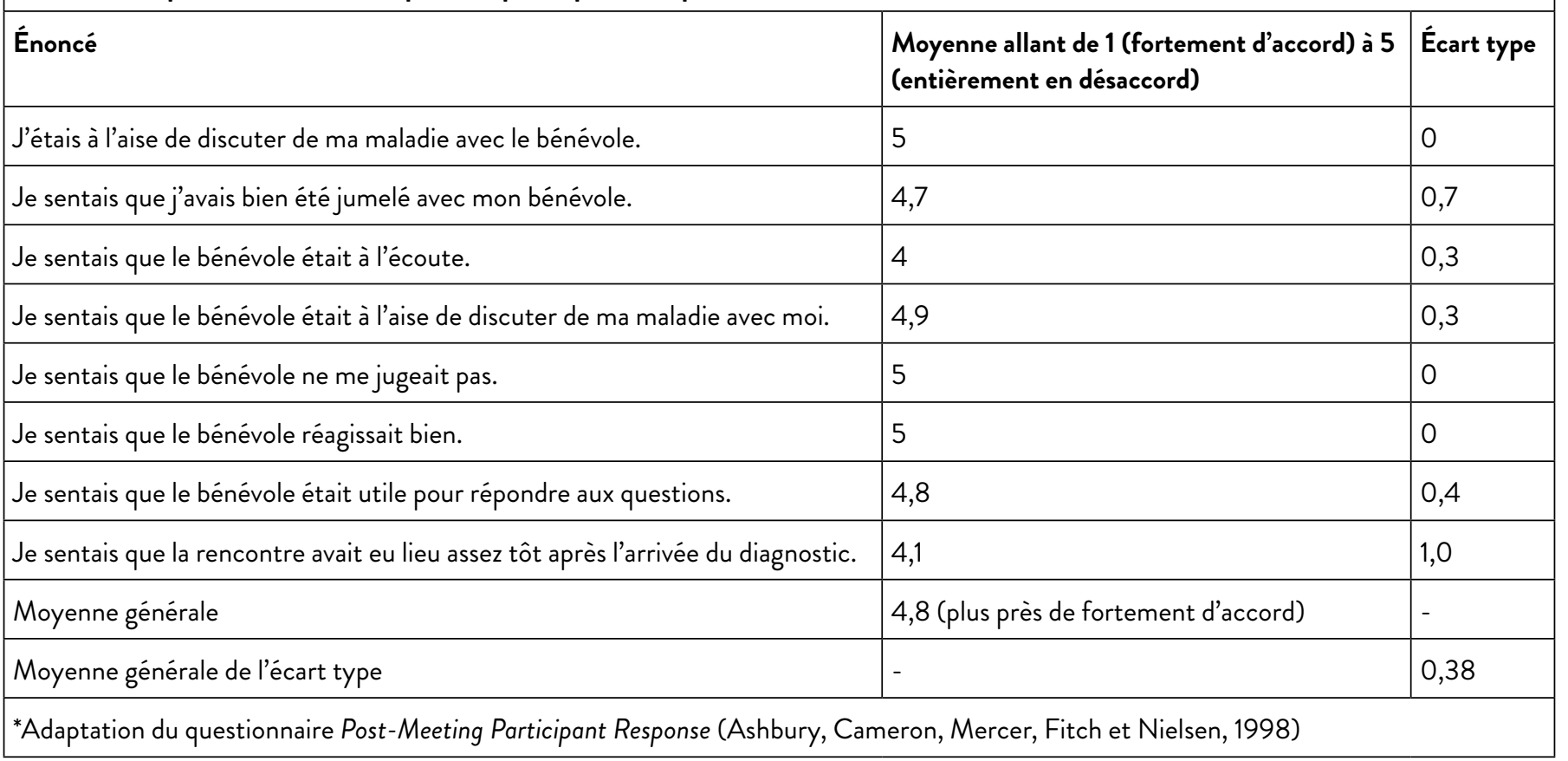




\section{Réponses aux questions de classement}

Pendant le premier entretien, on a demandé aux nouveaux patients participants de répondre à la question « Dans l'ensemble, à quel point cette rencontre a-t-elle été utile pour vous? » sur une échelle de 1 (《 d'aucune aide ») à 4 (" très utile »). La moyenne des réponses était de 3,35 (avec un écart type de 0,57 ), le plus près de « assez utile ». On a également posé la question suivante aux nouveaux patients participants: « En général, à quel point cette rencontre s'est-elle avérée négative pour vous? ». Sur une échelle de 1 (« pas du tout négative ») à 4 (« très négative »), la moyenne des réponses a été de 1,15 (écart type de 0,38 ), le plus près de « pas du tout négative ».

Pendant le deuxième entretien (environ six semaines après la rencontre avec le bénévole), on a encore posé cette question aux nouveaux patients participants: «Dans l'ensemble, à quel point cette rencontre a-t-elle été utile pour vous? ». La moyenne des réponses a été de 2,9 (écart type de o,86), le plus près de «assez utile ». Pour terminer, on a encore posé la question suivante aux nouveaux patients participants: « En général, à quel point cette rencontre s'est-elle avérée négative pour vous? ». La moyenne des réponses a été de 1,05 (écart type de 0,15$)$, le plus près de « pas du tout négative ».

\section{Réponses aux questions fermées}

Pendant le premier entretien, on a posé de nombreuses questions fermées aux nouveaux patients participants.

- En réponse à la question « Est-ce qu'une rencontre avec le bénévole a été suffisante pour vous ou souhaiteriez-vous des rencontres de suivi? » (si le patient souhaitait davantage de rencontres: «Combien de rencontres supplémentaires souhaiteriez-vous? ») :

- Quatre participants (40\%) ont dit qu'une rencontre leur suffisait; trois participants $(30 \%)$ ont dit qu'ils souhaiteraient une rencontre de suivi; un participant (10\%) a dit qu'il souhaiterait deux rencontres de suivi; un participant (10\%) a dit qu'il souhaiterait trois rencontres de suivi; et un participant $(10 \%)$ a dit qu'il souhaiterait un nombre illimité de rencontres.

- En réponse à la question « Sentez-vous qu'il était important que votre bénévole soit un survivant d'une tumeur cérébrale au lieu d'un survivant à un autre type de cancer? » :

- Les dix participants (100\%) ont répondu par l'affirmative.

- En réponse à la question « Sentez-vous que votre partenaire (ou une autre personne de soutien clé) trouverait utile d'avoir une rencontre similaire avec un bénévole qui a déjà apporté du soutien à un survivant d'une tumeur cérébrale? »:

- Huit participants $(80 \%)$ ont répondu « Oui »; un participant (10\%) a répondu « Non », en raison d'une barrière linguistique; et un participant (10\%) a dit que ce n'était pas pertinent, car il n'avait pas de personne de soutien clé.

- Pendant l'entretien de suivi, on a posé aux nouveaux patients participants la question « Votre rencontre avec le bénévole, y compris tout ce dont vous avez discuté ou la brochure sur les ressources qu'il vous a transmise, a-t-elle eu des répercussions sur la manière dont vous avez eu accès aux ressources de soutien depuis lors? »:

- Un participant (10\%) a répondu « Oui ».

- Neuf participants (90\%) ont répondu « Non ».

\section{Réponses aux questions ouvertes}

Pendant le premier entretien, on a interrogé les nouveaux patients participants sur les sujets d'importance et les suggestions d'amélioration. Leurs réponses sont énumérées ci-dessous, accompagnées du compte de fréquence correspondant au nombre de participants qui ont mentionné chaque sujet.

- Sujets importants:

- Écouter les autres raconter leur histoire (3); s'ajuster aux changements de priorité de vie (3); gérer la transition de carrière (3); changements recommandés en matière de style de vie (2); stratégies d'adaptation spécifiques (2); traitement des effets secondaires (2); activité physique (2).

- Améliorations suggérées:

- Davantage de structure (3); rencontre tenue plus tôt après la tombée du diagnostic (3); plus de renseignements personnels divulgués par le bénévole (2); des rencontres plus longues (2).

Avantages indiqués par les nouveaux patients participants

En ce qui concerne les avantages signalés par les nouveaux patients participants, le procédé de retriage et d'amélioration par thème a été effectué par trois codeurs naïf jusqu'à ce qu'un coefficient Kappa de 0,701 soit atteint. Au total, sept thèmes liés aux avantages ont été établis. Seuls les cinq thèmes qui ont été abordés par au moins quatre nouveaux patients participants sont mentionnés ici (voir tableau 4). Le retriage et l'amélioration en ce qui a trait aux sous-thèmes (seulement effectués dans le sous-thème « espoir accru ») ont été cessés après que le codage ait été effectué à l'aide d'un seul codeur naiff, parce qu'un coefficient Kappa de 0,745 avait été atteint.

Espoir accru. La forme la plus puissante d'avantage qui a ressorti des données a été l'augmentation de l'espoir, neuf des dix nouveaux patients participants ayant émis au moins une affirmation à cet effet. Quatre sous-thèmes distincts sont apparus plus clairement à partir de ce thème.

Tableau 4 : Certains thèmes liés aux avantages indiqués par les nouveaux patients participants

\begin{tabular}{|l|l|}
\hline Thème & $\begin{array}{l}\text { Nombre de nouveaux } \\
\text { patients participants qui } \\
\text { ont indiqué le thème }\end{array}$ \\
\hline Espoir accru & 9 \\
\hline Conseils précieux & 7 \\
\hline Savoir ce qu'il en est vraiment & 6 \\
\hline Combattre la solitude & 5 \\
\hline Prendre conscience de ce qui importe & 4 \\
\hline
\end{tabular}

1) Le premier sous-thème lié à l'espoir était « avoir une espérance de vie plus longue que prévu ». Huit des nouveaux patients participants ont exprimé le sentiment que, grâce à la rencontre avec les bénévoles, ils ressentaient davantage l'espoir de vivre plus longtemps que ce qu'ils avaient cru euxmêmes possible. Cet espoir accru émanait du fait de s'être 
assis avec un survivant d'une tumeur cérébrale qui allait bien, ce qui leur a fourni une preuve tangible que ce résultat était possible après le traitement. Mona a raconté:

"Je ne me permettais aucune sorte d'avenir, je ne me permettais pas de dire: "L'an prochain, je vais faire ceci." Je me restreignais moi-même; cétait presque comme si je dressais une barrière autour de moi... Puis Mike m'a donné de l'espoir... Je ne pensais même pas jusqu'à Noël... J'ai plus d'espoir maintenant que j'en avais (d'être encore vivante à Noël)... C'est de là que me vient l'espoir. Parce que lorsqu'on n'a plus d'espoir et qu'on est malade, le bonheur de vivre, le bien-être, tout change. »

2) Le deuxième sous-thème lié à l'espoir était « l'espoir, inspiré par l'exemple du bénévole, de vivre malgré le diagnostic ». Six des nouveaux patients participants ont affirmé que l'exemple de réussite personnelle des patients intervenants bénévoles confrontés aux difficultés de vivre avec une tumeur cérébrale les a encouragés à croire qu'un résultat similaire était possible pour eux. Mona a raconté:

«Et les choses qu'il a accomplies depuis qu'il a été diagnostiqué - il a voyagé, il a eu un enfant; c'est vraiment merveilleux. Ça me donne de l'espoir... L'espoir d'atteindre un état comme le sien.»

3) Le troisième sous-thème lié à l'espoir était « l'espoir d'une meilleure guérison que ce qu'on avait craint ». Trois des nouveaux patients participants ont trouvé que la rencontre avec les patients intervenants bénévoles avait favorisé un sentiment d'espoir selon lequel les répercussions médicales de leur maladie ne provoqueraient pas une baisse grave de leur qualité de vie, comme ils l'avaient craint. Gerry a indiqué:

«... On se fait une image des gens qui sont aux prises avec une déficience mentale en raison d'une intervention chirurgicale... On croit qu'ils vont être comme Jack Nicholson à la fin de «Vol au-dessus d'un nid de coucou» et qu'il ne leur restera plus rien, ou qu'ils seront totalement différents. Alors il est bon de savoir, juste après une rencontre avec Mike, que ça ne l'a pas changé. »

4) Le dernier sous-thème lié à l'espoir était « l'espoir qui peut être transmis aux êtres chers ». Deux nouveaux patients participants ont dit que la rencontre leur avait fourni une sorte de mécanisme pour restaurer l'espoir de leurs êtres chers, qui étaient également très anxieux à la suite du diagnostic. Gerry a indiqué:

"Je suis plus inquiet pour mon épouse et aussi pour mes parents qui sont âgés, plus que je ne le suis pour moi-même en un sens; j'ai pu leur parler de l'état de Mike, et je pense que ça les a réconfortés.»

Conseils précieux. Sept des nouveaux patients participants ont exprimé qu'ils avaient tiré avantage des recommandations concrètes émises par leur patient intervenant bénévole. Gerry a dit:

"Je me rappelle quand Mike a raconté qu'il recevait des soins de massothérapie et d'acupuncture en raison du stress qu'il vivait et que ça l'aidait à se sentir mieux. Ça m'avait marqué... »
Savoir ce qu'il en est vraiment. Six des nouveaux patients participants ont trouvé encourageant et démystifiant de connaître de nombreuses expériences de la vie au quotidien avec une tumeur cérébrale, particulièrement en ce qui concerne les effets du traitement et de la tumeur au fil du temps. Plusieurs de ces nouveaux patients participants, qui avaient même cru que leur propre expérience liée à la tumeur serait unique et donc inévitablement différente de celles des patients intervenants bénévoles, ont trouvé utile d'avoir une image plus claire des problèmes auxquels ils pouvaient s'attendre. Alan a indiqué:

« D'en entendre parler par quelqu'un qui est passé par là plutôt que par un médecin qui nous dit "Vous pourriez ressentir ceci ou cela”... Je veux dire, j'aime mieux entendre quelqu'un dire "C'est ce qui m'est réellement arrivé», vous savez? C'est plus concret... »

Combattre la solitude. La moitié des nouveaux patients participants ont fait des témoignages qui illustraient un plus grand sentiment de rapprochement et ont partagé leur expérience après la rencontre avec les patients intervenants bénévoles. Certains ont dit qu'ils s'étaient sentis envahis par une solitude existentielle qui les avait troublés depuis l'arrivée du diagnostic. Gerry a affirmé:

«... J'ai trouvé ça utile parce que... lorsqu'on l'apprend, c'est comme si... Comme si quelque chose s'édifiait entre nous et le reste du monde, comme un mur de plexiglas... Alors ça fait du bien de parler avec des personnes comme Mike... Que ces personnes le ressentent de cette manière ou non, elles sont en quelque sorte une joyeuse bande de camarades. »

Au cours du même échange, Mona affirmait:

«... On se sent seuls à l'intérieur, et on a beau avoir beaucoup de personnes merveilleuses autour de nous qui nous offrent du soutien et nous aiment, cela n'arrive jamais à combler notre vide intérieur. Et il (le bénévole) se sentait pareil... C'est comme un baume sur une plaie.»

Prendre conscience de ce qui importe. Quatre nouveaux patients participants ont raconté que les rencontres avec les patients intervenants bénévoles les avaient éveillés au fait d'accorder la priorité à ce qui comptait le plus dans leur vie. Gerry, qui est un amoureux de la musique de longue date, mais qui effectuait des études en gériatrie au moment du diagnostic, a raconté:

«(Le patient intervenant bénévole) m’a dit qu'une place l'attendait dans un cabinet juridique, mais qu'il n'était pas certain de vouloir encore pratiquer le droit... Cela a sonné une cloche... Je veux dire que lorsqu'on sait que le temps est limité, on veut utiliser ce temps à faire ce qu'on aime vraiment. »

\section{Conséquences négatives rapportées par les nouveaux patients participants}

Deux événements indésirables ont été rapportés par les nouveaux patients participants. Dans un des cas, Gerry, un nouveau patient participant, a été surpris d'apprendre qu'on avait donné à son patient intervenant bénévole une estimation 
de son espérance de vie, alors que Gerry n'en avait pas reçue. Ceci a amené le patient intervenant bénévole de Gerry à faire des spéculations sur le fait que la raison « réelle » pour laquelle l'équipe médicale de Gerry ne lui avait pas donné de pronostic concret était que la pratique médicale changeait et que les oncologues n'avaient plus l'impression qu'il était utile pour les patients d'obtenir une durée d'espérance de vie. En réponse à cette spéculation, Gerry a indiqué:

«... C'est là peut-être un point négatif que j'ai retiré de l'expérience... cette question des cinq ans. Je m'en souviens encore, c'est ce qu'on lui avait dit. »

Il est explicitement déconseillé aux patients intervenants bénévoles d'interpréter ou d'expliquer des opinions et des avis émis par les médecins, car cela peut mener à des évaluations inexactes. De plus, cela peut provoquer de l'anxiété chez les nouveaux patients participants et miner leur confiance envers l'intégrité de leur équipe médicale. Néanmoins, cet événement semble avoir eu peu d'impact sur Gerry, étant donné qu'une semaine après l'intervention, il a accordé une note de seulement 1,5 sur 4 pour les effets négatifs, comparativement à une note de 3 sur 4 pour les effets positifs découlant de l'entretien.

Le deuxième événement indésirable concernait Shirley, une nouvelle patiente participante, qui a cru percevoir que son patient intervenant bénévole était devenu émotif plusieurs fois pendant leur rencontre, et que, pendant ces épisodes, il avait changé l'orientation de la rencontre de manière inappropriée pour l'axer vers ses propres besoins émotionnels. Cela a fait en sorte que Shirley a quitté la rencontre avec un sentiment négatif, tel qu'elle l'a indiqué dans ses commentaires :

«... Il semblait submergé d'émotion... Je me suis sentie un peu mal, car je sentais que les rôles étaient inversés... Et qu'il me faisait un peu perdre mon temps par moments... »

Toutefois, les répercussions de cet événement semblent n'avoir eu qu'un léger effet sur Shirley, tel que mis en évidence par sa note initiale de 2 sur 4 pour les effets négatifs, comparativement à 3 sur 4 pour les effets positifs de la rencontre.

\section{Avantages indiqués par les patients intervenants bénévoles}

De façon collective, les deux bénévoles ont mis en relief trois avantages spécifiques qu'ils avaient retirés de leur participation à cette étude.

Augmentation du sentiment de fierté et de positivisme. Les deux patients intervenants bénévoles ont dit que d'avoir le sentiment de guider les patients nouvellement diagnostiqués les avait aidés à avoir une meilleure estime d'eux-mêmes et à retirer du positivisme au sujet de leur propre expérience. Yves a dit:

«... Ça fait du bien d'avoir ce type de répercussions sur les autres... Pour moi, ça n'aurait pas de sens d'avoir survécu et puis d'oublier, et de ne pas pouvoir servir de levier pour les autres. Cela donne donc un sens à la poursuite de ma croissance, pour ainsi dire, et à la prise en compte de mon expérience.»

Mike a exprimé un sentiment similaire:
«J'en retire beaucoup d'avantages énormes... Je me sens vraiment très bien après les rencontres. J'ai l'impression d'avoir donné de l'espoir à un patient... »

Diminution de l'anxiété. Mike était d'avis que le bénévolat l'avait aidé à renforcer sa confiance envers son habileté à bien gérer les aspects stressants de sa maladie:

"Il y a une grande variété de facteurs, mais depuis le début de ces rencontres, mon anxiété a diminué de manière extraordinaire. Je ne fais presque plus d'anxiété au sujet des effets secondaires. Je ne suis plus, ou presque plus, anxieux au sujet de mes IRM. Je pense que ça m'a simplement permis de prendre du recul... »

Mike a également fait observer que sa participation au programme de bénévolat l'avait aidé à atteindre un plus grand sentiment de paix et de sérénité, même s'il est confronté à la crainte de sa propre mort:

«La mort fait continuellement partie de mes pensées, peu importe ce que je suis en train de faire... Que je sois en train de jouer à des jeux vidéo, de m'entraîner ou de faire autre chose... Mais lorsque je suis en présence d'un autre patient atteint d'une tumeur cérébrale au stade précoce, c'est là que je me rappelle toute la peur que j'avais. Je me dis alors que je peux surmonter la situation... Je dois enlever mon masque. Et je crois que le fait d'être authentique m'aide vraiment. Tant de fois j'ai affiché un air courageux, mais au fond de moi j'avais peur et j'étais anxieux. »

Amélioration des aptitudes en matière de communication. Yves a eu le sentiment que la formation l'avait aidé à devenir plus réfléchi et un meilleur communicateur, particulièrement au travail.

\section{Conséquences indésirables signalées par les patients intervenants bénévoles}

Interrogé à ce sujet, Yves n’a indiqué aucune conséquence indésirable comme résultat du bénévolat. Pour sa part, Mike a identifié une seule et minime conséquence indésirable. Au début du projet, Mike trouvait que les discussions tenues au sujet des crises avec les nouveaux patients participants le rendaient anxieux à l'idée d'avoir des crises même s'il n'en avait pas. Cependant, cette anxiété a rapidement diminué et cessé grâce à du soutien et à de la supervision.

\section{Difficultés signalées par les patients intervenants bénévoles}

Les deux patients intervenants bénévoles ont exprimé une confiance générale envers leur capacité à gérer les rencontres efficacement et leur capacité à réguler leurs propres émotions afin de mieux soutenir les nouveaux patients. Mike a toutefois indiqué une difficulté à ce sujet, comme le montre son commentaire ci-dessous:

" Je suis parfois devenu émotif lorsque je pensais à ma conjointe et à mon fils, et généralement les hommes plus âgés intervenaient et essayaient de me réconforter, mais je devais dire: "Cette rencontre est pour vous et elle doit porter sur vous." ... Cela semblait remettre la rencontre dans la bonne direction.» 


\section{Réactions des patients intervenants bénévoles au sujet de la formation, du soutien et de la supervision}

Les deux patients intervenants bénévoles se sont montrés très satisfaits au sujet de la formation, du soutien et de la supervision qu'ils ont reçus pour leur rôle de bénévole, et ils ont particulièrement aimé l'aspect expérientiel des jeux de rôle de la formation.

\section{DISCUSSION}

Cette étude comprenait l'élaboration et la mise en place d'une intervention de soutien individuelle par les pairs pour les patients nouvellement diagnostiqués d'une tumeur cérébrale primitive. Les patients qui avaient déjà terminé un traitement initial pour une tumeur cérébrale ont reçu une formation pour offrir du soutien et de l'information aux nouveaux patients.

Le résultat le plus important qui est ressorti de l'étude est que l'intervention s'est avérée bénéfique pour les nouveaux patients participants et n'a causé aucun effet indésirable majeur. Cette conclusion est soutenue par les deux notations quantitatives de l'intervention et par les observations éloquentes des nouveaux patients participants au sujet des résultats positifs. Ce résultat souligne l'importance du programme de soutien par les pairs pour les patients nouvellement diagnostiqués d'une tumeur cérébrale primitive et renforce également la conclusion obtenue par les chercheurs précédents (Macvean, White et Sanson-Fischer, 2008) au sujet de la valorisation des programmes de soutien individuel par les pairs pour les patients cancéreux de manière plus générale. Ce résultat est particulièrement fondamental à la lumière des résultats d'une revue de la documentation sur les besoins en soins psychosociaux et de soutien des patients atteints d'un gliome (Ford, Catt, Chalmers et Fallowfield, 2012), qui ont révélé que les patients n'étaient généralement pas satisfaits de la communication avec leurs prestataires de soins de santé. Les raisons derrière cette insatisfaction étaient de natures variées et comprenaient une absence de messages positifs et des lacunes dans la préparation des patients pour la vie après leur traitement. Les nouveaux patients participants qui ont bénéficié de notre intervention dans le cadre de l'étude ont explicitement mentionné les avantages de rencontrer des patients intervenants bénévoles; ces derniers, ayant survécu à la maladie, ont joué un rôle de modèle positif et les ont menés à avoir une attitude plus optimiste au sujet de la vie après le traitement.

En plus d'être bénéfique pour les nouveaux patients, l'intervention a aussi eu des résultats positifs pour les « anciens » patients, toujours sans causer aucun effet négatif important. Ce résultat est cohérent avec les récentes données probantes selon lesquelles les patients atteints de cancer retirent des avantages sur le plan psychologique à titre de pairs bénévoles (Pistrang, Jay, Gessler et Barker, 2013), mais il contraste avec une étude précédente qui n’a pas conclu que ceci était le cas (Giese-Davis et al., 2006).

Le fait que nos patients intervenants bénévoles aient retiré des avantages de cette expérience, même si ces rencontres les obligeaient à être confrontés à leurs propres craintes existentielles, indique que l'effort mis dans le soutien apporté aux autres peut avoir favorisé un sentiment accru de maîtrise de soi. Cette hypothèse est cohérente avec la recherche, indiquant que l'exposition contrôlée constitue un puissant moyen de diminuer l'anxiété associée à des situations très lourdes au point de vue psychologique (Hayes, Strosahl, Wilson, Bissett, Pistorello, Toarmino et al., 2004).

La brièveté de l'intervention mérite de faire l'objet de discussions, parce que les avantages indiqués ont été constatés même si l'intervention ne comprenait qu'une seule rencontre. Ce résultat correspond à ceux déjà mentionnés concernant le programme «Surmonter le cancer » (Dunn, Stegingia, Occhipinti et Wilson, 1999) et également aux effets inattendus possibles d'une seule séance de psychothérapie (Bloom, 2001). Parallèlement, la majorité des nouveaux patients participants ont toutefois exprimé qu'ils auraient préféré avoir au moins une rencontre de suivi. Ainsi, les itérations futures de cette intervention devraient comprendre la possibilité d'une rencontre de suivi pour les nouveaux patients qui le souhaitent.

Il importe également de noter que, même si les nouveaux patients participants ont retiré des avantages de l'intervention, il y a eu une augmentation très limitée dans l'adoption subséquente d'autres mesures de soutien par les nouveaux patients participants (seulement $10 \%$ des nouveaux patients participants étaient d'accord pour dire que les rencontres avaient eu des conséquences sur la façon dont ils avaient accédé aux ressources de soutien dans les six semaines suivantes). Ce résultat est contraire à nos attentes selon lesquelles le fait d'avoir une interaction positive avec des pairs où l'on offre de l'information sur les ressources pertinentes en matière de soutien aux nouveaux patients participants améliorerait leur recherche subséquente de ressources. Il est possible qu'une simple interaction avec un patient intervenant bénévole n'ait pas suffi à augmenter la motivation des nouveaux patients participants à chercher d'autres formes de soutien. D'un autre côté, peutêtre que cette intervention a mené à une augmentation dans l'adoption subséquente d'autres services de soutien par les nouveaux patients participants, mais après notre brève période de suivi de six semaines. Finalement, le fait détablir à quel point ce bref type d'intervention bénévole peut inciter les patients nouvellement diagnostiqués d'une tumeur cérébrale à accéder plus activement aux services de soutien constitue une question importante pour la recherche future.

Dans un autre ordre d'idées, presque tous les nouveaux patients participants ont affirmé avoir l'impression qu'une intervention similaire aurait été utile pour leur conjoint ou leur soignant clé. Nos conclusions indiquent que les soignants des personnes nouvellement diagnostiquées d'une tumeur cérébrale, qui portent également un lourd fardeau d'anxiété, de détresse et d'incertitude (Janda, Steginga, Dunn, Langbecker, Walker et Eakin, 2008), peuvent aussi retirer des avantages des rencontres avec d'anciens soignants qui ont fait face à ces épreuves avec succès. Ceci s'avère particulièrement pertinent du point de vue des possibles déficiences cognitives et physiques des patients atteints d'une tumeur cérébrale primitive, ainsi que de l'importance des soignants familiaux en ce qui a trait à la prise de décisions et à la prestation des soins au patient. 
Enfin, l'importance de l'engagement du patient dans la mise en place de programmes de soins en neuro-oncologie ne peut pas être ignorée. Cette étude démontre que les membres de notre CCPF sont d'un apport inestimable en ce qui concerne les besoins des patients atteints d'une tumeur cérébrale et la prestation de conseils au sujet des manières les plus efficaces de répondre à ces besoins.

\section{Limites}

Cette recherche comporte de nombreuses limites qui se répercutent sur sa validité interne et sa capacité à être généralisée. Létude a nécessité l'élaboration d'un programme de formation pour les patients intervenants bénévoles, a été effectuée par un conseiller en recherche et a requis un niveau important d'efforts et de supervision du personnel. Or, ce programme pourrait ne pas convenir aux centres de traitement du cancer qui possèdent moins de ressources. Cela pourrait demeurer vrai même si le nombre d'heures de formation et de supervision pour cette intervention est diminué sans rien enlever à son efficacité ou à son innocuité.

Dans cette même perspective, nous avons pu recruter deux patients intervenants bénévoles exceptionnels. En fait, un de nos patients intervenants bénévoles s'est montré particulièrement intéressé par le counseling et avait commencé un programme de formation dans ce domaine. Le personnel des autres centres pourrait trouver difficile de recruter des bénévoles aussi hautement qualifiés et motivés pour participer à un programme de cette nature.

Tous les nouveaux patients participants et les patients intervenants bénévoles ont été les patients de la deuxième auteure et ont également reçu une attention spéciale du premier auteur pendant le programme. Dans ces conditions, il est possible que ces relations puissent être la cause d'une exagération de la part des nouveaux patients participants ou des patients intervenants bénévoles en ce qui a trait aux avantages découlant de l'intervention.

Nos critères d'inclusion ont restreint l'accès à l'intervention aux patients qui étaient au mieux sur le plan médical et qui parlaient anglais. De plus, à l'exception d'un, tous les nouveaux patients participants étaient de race blanche. Ces facteurs diminuent la représentativité de notre échantillon et, ce faisant, diminuent le potentiel de généralisation de nos résultats.

L'intervention a été menée par deux patients intervenants bénévoles seulement et, pour cette raison, les résultats auraient pu être différents si d'autres patients intervenants bénévoles y avaient participé.

Finalement, même si des efforts ont été mis pour attester de la fiabilité entre les codeurs, il est possible que les thèmes qui ont émergé de l'analyse qualitative puissent avoir été différents entre les mains d'une autre équipe de recherche.

\section{Résumé}

Les patients intervenants dûment formés et atteints d'une tumeur cérébrale peuvent être d'un soutien important pour les patients nouvellement diagnostiqués. Le sentiment de jouer un rôle utile et de modèle peut également représenter un avantage pour les patients qui ont déjà souffert d'une tumeur cérébrale et favoriser un sentiment d'auto-efficacité, d'intégrité et de cohérence dans une vie ébranlée par une maladie dévastatrice. Ce modèle de soutien correspond à un intérêt croissant dans la défense des droits et des intérêts du patient ainsi que dans l'autodétermination du patient et peut répondre aux besoins non comblés pour l'amélioration de la communication et du soutien. Les résultats de ce programme pilote sont préliminaires, mais ils indiquent qu'il serait important de mener d'autres recherches en matière de soutien par les pairs chez les patients atteints d'une tumeur cérébrale ainsi que chez les membres de leur famille.

\section{Annexe A: Questions d'entretien sélectionnées}

Questions ouvertes pour les nouveaux patients participants

- La rencontre avec le bénévole a-t-elle été utile?

- Dans l'affirmative, de quelle manière?

- La rencontre avec le bénévole a-t-elle été une expérience négative pour vous, de quelque manière que ce soit?

- Dans l'affirmative, de quelle manière?

- Y avait-il des sujets de discussion qui ont été particulièrement utiles pour vous?

- Avez-vous des suggestions d'améliorations à apporter au programme?

Questions ouvertes pour les patients intervenants bénévoles

- Avez-vous retiré des avantages de cette expérience? Dans l'affirmative, de quelle manière?

- Jouer ce rôle a-t-il été une expérience négative pour vous, de quelque manière que ce soit? Dans l'affirmative, de quelle manière?

- Quelles difficultés avez-vous rencontrées au sujet de l'animation des rencontres?

- À quel point la formation vous a-t-elle préparé à jouer ce rôle?

- À quel point le personnel vous a-t-il soutenu? 


\section{RÉFÉRENCES}

Altman, D.G. (1991). Practical statistics for medical research. Londres, RU: Chapman and Hall.

Ashbury, F.D., Cameron, C., Mercer, S.L., Fitch, M. et Nielsen, E. (1998). One-on-one peer support and quality of life for breast cancer patients. Patient Education and Counseling, 35, 89-100.

Bloom, B.L. (2001). Focused single-session psychotherapy: A review of the clinical and research literature. Brief Treatment and Crisis Intervention, 1, 75-86.

Dunn, J., Steginga, S.K., Occhipinti, S. et Wilson, K. (1999). Evaluation of a peer support program for women with breast cancer - Lessons for practitioners. Journal of Community and Applied Social Psychology, 9, 13-22.

Ford, E., Catt, S., Chalmers, A. et Fallowfield, L. (2012). Systematic review of supportive care needs in patients with primary malignant brain tumours. Neuro-oncology, 14(4), 392-404.

Giese-Davis, J., Bliss-Isberg, C., Carson, K., Star, P., Donaghy, J., Cordova, M.J., Stevens, N., ... Spiegel, D. (2006). The effect of peer counseling on quality of life following diagnosis of breast cancer: An observational study. Psycho-oncology, 15, 1014-1022.

Grande, G.E., Myers, L.B. et Sutton, S.R. (2006). How do patients who participate in cancer support groups differ from those who do not? Psycho-Oncology, 15, 321-334.

Hagopian, G.A. (1993). Cognitive strategies used in adapting to a cancer diagnosis. Oncology Nursing Forum, 20(5), 759-763.

Hayes, S.C., Strosahl, K.D., Wilson, K.G., Bissett, R.T., Pistorello, J., Toarmino, D. et al. (2004). Measuring experiential avoidance: A preliminary test of a working model. The Psychological Record, 54, $553-578$.

Hoey, L.M., Leropoli, S.C., White, V.M. et Jefford, M. (2008). Systematic review of peer-support programs for people with cancer. Patient Education and Counseling, 70(3), 315-337.
Janda, M., Steginga, S., Dunn, J., Langbecker, D., Walker, D. et Eakin, E. (2008). Unmet supportive care needs and interest in services among patients with a brain tumour and their carers. Patient Education and Counseling, 71, 251-258.

Macvean, M.L., White, V.M. et Sanson-Fisher, R. (2008). One-to-one volunteer support programs for people with cancer: A review of the literature. Patient Education and Counseling, 70(1), 10-24.

Pelletier, G., Verhoef, M.J., Khatri, N. et Hagen, N. (2002). Quality of life in brain tumor patients: The relative contributions of depression, fatigue, emotional distress, and existential issues. Journal of Neurooncolog $\gamma, 57,41-49$.

Pistrang, N., Jay, Z., Gessler, S. et Barker, C. (2013). Telephone peer support for women with gynaecological cancer: Benefits and challenges for supporters. Psycho-Oncology, 22, 886-894.

Sherman, A.C., Pennington, J., Simonton, S., Latif, U., Arent, L. et Farley, H. (2008). Determinants of participation in cancer support groups. International Journal of Behavioral Medicine, 15, 92-100.

Strauss, A. et Corbin, J. (1990). Basics of qualitative research: Grounded theory procedures and techniques. Newbury Park, CA: Sage.

Taphoorn, M.J., Sizoo, F. et Bottomly, A. (2010). Review on quality of life issues in patients with primary brain tumours. Oncologist, 15, 618-626.

Vilhauer, R.P. (2009). Perceived benefits of online support groups for women with metastatic breast cancer. Women Q Health, 49(5), 381-404.

Wellisch, D., Kaleita, T., Freeman, D., Cloughesy, T. et Goldman, J. (2002). Predicting major depression in brain tumor patients. Psycho-Oncology, 11, 230-238.

Wimmer, R.D. et Dominick, J.R. (1991). Mass media research: An introduction, $3^{\mathrm{e}}$ éd., Belmont, CA: Wadsworth. 